\title{
Capacitance fluctuations causing channel noise reduction in stochastic Hodgkin-Huxley systems
}

\author{
G Schmid, I Goychuk and P Hänggi \\ Institut für Physik, Universität Augsburg, D-86135 Augsburg, Germany \\ E-mail: Gerhard.Schmid@physik.uni-augsburg.de
}

Received 20 September 2006

Accepted for publication 3 November 2006

Published 22 November 2006

Online at stacks.iop.org/PhysBio/3/248

\begin{abstract}
Voltage-dependent ion channels determine the electric properties of axonal cell membranes. They not only allow the passage of ions through the cell membrane, but also contribute to an additional charging of the cell membrane resulting in the so-called capacitance loading. The switching of the channel gates between an open and a closed configuration is intrinsically related to the movement of gating charge within the cell membrane. At the beginning of an action potential, the transient gating current is opposite to the direction of the current of sodium ions through the membrane. Therefore, the excitability is expected to become reduced due to the influence of a gating current. Our stochastic Hodgkin-Huxley-like modeling takes into account both the channel noise-i.e. the fluctuations of the number of open ion channels - and the capacitance fluctuations that result from the dynamics of the gating charge. We investigate the spiking dynamics of membrane patches of a variable size and analyze the statistics of the spontaneous spiking. As a main result, we find that the gating currents yield a drastic reduction of the spontaneous spiking rate for sufficiently large ion channel clusters. Consequently, this demonstrates a prominent mechanism for channel noise reduction.
\end{abstract}

\section{Introduction}

Following the study of Hodgkin and Huxley [1], most of the models of axons have treated the generation and propagation of action potentials using deterministic differential equations. Since the work of Lecar and Nossal [2] it has become increasingly evident, however, that not only the synaptic noise but also the randomness of the ion channel gating itself may cause threshold fluctuations in neurons [3, 4]. Therefore, channel noise which originates in the stochastic nature of the ion channel dynamics should be taken into account [3, 4]. For example, in mammalian ganglion cells both the synaptic noise and the channel noise might equally contribute to the neuronal spikes variability [5]. Due to a finite size, the origin of the channel noise is basically due to fluctuations of the mean number of open ion channels around the corresponding mean values. Therefore, the strength of the channel noise is mainly determined by the number of ion channels participating in the generation of action potentials. Channel noise impacts, for example, such features as the threshold to spiking and the spiking rate itself [6-12], the anomalous noiseassisted enhancement of transduction of external signals, i.e. the phenomenon of stochastic resonance [13-24], and the efficiency for synchronization [25]. Interestingly enough, there exist optimal patch sizes for which the spike production becomes more regular, or the response to external stimuli optimizes [15, 16, 21-23, 26]. The objective of this work is to investigate the spontaneous spiking of cell membrane patches when gating charge effects are also considered.

When an ion channel opens or closes, an effective gating charge is moved across the membrane [27]. This motion creates the so-called gating current which is experimentally measurable [28-30]. The influence of gating currents was not explicitly considered in the original Hodgkin-Huxley (HH) model [1]. In 1975 Hodgkin [31] and, independently, Adrian [32], firstly inquired theoretically into the influence of the ion channel density on the velocity of the action potential propagation along the squid giant axon by taking into consideration the gating currents of sodium ion channels (via an effective capacitance loading caused by ion channels, 
see also [33]). Remarkably, they found an optimal ion channel density for which the signal velocity is maximal [31, 32, 3436]. This led to the emergence of the Hodgkin's maximum velocity hypothesis, i.e. that the living species can adopt optimal densities of ion channels to maximize the rate of neuronal information transduction.

In this work we investigate the influence of both the gating currents and the channel noise on the spontaneous spiking. At the initiation of a spike, there occur two competitive currents in the presence of ion channels. First, an ionic current, which is caused by the influx of positively charged sodium ions through open sodium ion channels into the cell, and second, a gating current that flows in the opposite direction during the transition of sodium ion channels from closed to open state. The latter one is due to the movement of the positive gating charge from the inside of the cell membrane to the outside. This current actually precedes the first one, and terminates when the channel is in its open state. However, due to spontaneous fluctuations between the channel's states, a stochastic component of gating current also emerges. Moreover, there are also gating currents due to potassium channels which have been neglected within the macroscopic, noise-free model [31, 32, 34, 35]. These polarization currents of membrane-bound charges can substantially contribute to the overall polarization current across the membrane, which can be considered as an electrical capacitor. Therefore, the influence of gating currents on the excitability of small ion channel clusters is a priori not clear and necessitates further research.

In section 2 we show how channel noise can be taken into account within a Langevin equation approach. Section 3 deals with the extensions to the standard $\mathrm{HH}$ model with respect to gating charge effects. We discuss the influence of gating charge on the dynamics with respect to excitability due to a constant stimulus and as well as on the deterministic spiking period. Thenceforth, in section 4 we consider the situation where both channel noise and gating charge are considered and demonstrate the influence on the mean interspike interval. The effect of intrinsic coherence resonance in the presence of gating currents is discussed in section 5 .

\section{A stochastic generalization of the Hodgkin-Huxley model}

According to the standard $\mathrm{HH}$ model, the dynamics of the membrane potential $V$ follows

$$
\begin{gathered}
C \frac{\mathrm{d}}{\mathrm{d} t} V+G_{\mathrm{K}}(n)\left(V-E_{\mathrm{K}}\right)+G_{\mathrm{Na}}(m, h)\left(V-E_{\mathrm{Na}}\right) \\
\quad+G_{\mathrm{L}}\left(V-E_{L}\right)=I_{\mathrm{ext}}(t),
\end{gathered}
$$

with the potassium and sodium conductances given by

$$
G_{\mathrm{K}}(n)=g_{\mathrm{K}}^{\max } n^{4}, \quad G_{\mathrm{Na}}(m, h)=g_{\mathrm{Na}}^{\max } m^{3} h .
$$

In equation (1), $V$ denotes the membrane potential measured throughout this work in $\mathrm{mV}$ and $C=1 \mu \mathrm{F} \mathrm{cm}^{-2}$ is the capacity of the cell membrane. The time $t$ is scaled in $\mathrm{ms}$. Furthermore, $E_{\mathrm{Na}}=50 \mathrm{mV}, E_{\mathrm{K}}=-77 \mathrm{mV}$ and $E_{\mathrm{L}}=-54.4 \mathrm{mV}$ are the reversal potentials for the sodium, potassium and leakage currents, respectively. The leakage conductance is assumed to be constant. $G_{\mathrm{L}}=0.3 \mathrm{mS} \mathrm{cm}^{-2}$, and $g_{\mathrm{K}}^{\max }=36 \mathrm{mS} \mathrm{cm}^{-2}$ and $g_{\mathrm{Na}}^{\max }=120 \mathrm{mS} \mathrm{cm}^{-2}$, respectively, denote the maximal potassium and sodium conductances, when all ion channels are open. $I_{\text {ext }}(t)$ indicates an external current stimulus. Equation (1) is nothing but a Kirchhoff law for an electrical circuit made of a membrane capacitor and variable nonlinear conductances assuming that the conductances of open ion channels are Ohmic, i.e. all the nonlinearity comes from their gating behavior.

The gating variables $n, m$ and $h$, cf equations (1) and (2), describe the mean ratios of the open gates of the specific ion channels. Assuming gate independence, the factors $n^{4}$ and $m^{3} h$ are the mean portions of the open ion channels within a membrane patch. The dynamics of the gating variables are determined by voltage-dependent opening and closing rates $\alpha_{x}(V)$ and $\beta_{x}(V)(x=m, h, n)$. Taken at $T=6.3{ }^{\circ} \mathrm{C}$, they read $[1,42,43]$

$$
\begin{gathered}
\alpha_{m}(V)=0.1 \frac{V+40}{1-\exp \{-(V+40) / 10\}}, \\
\beta_{m}(V)=4 \exp \{-(V+65) / 18\}, \\
\alpha_{h}(V)=0.07 \exp \{-(V+65) / 20\}, \\
\beta_{h}(V)=\frac{1}{1+\exp \{-(V+35) / 10\}}, \\
\alpha_{n}(V)=0.01 \frac{V+55}{1-\exp \{-(V+55) / 10\}}, \\
\beta_{n}(V)=0.125 \exp \{-(V+65) / 80\} .
\end{gathered}
$$

The dynamics of the mean fractions of open gates reduces in the standard $\mathrm{HH}$ model to relaxation dynamics:

$$
\frac{\mathrm{d}}{\mathrm{d} t} x=\alpha_{x}(V)(1-x)-\beta_{x}(V) x, \quad x=m, h, n .
$$

Such an approximation is valid for very large numbers of ion channels and whenever fluctuations around their mean values are negligible.

However, each channel defines in fact a bistable stochastic element which fluctuates between its closed and open states. The same is valid for the gates which are assumed to be independent in the $\mathrm{HH}$ model. The number of open gates undergoes a birth-and-death-like process. The corresponding master equation can readily be written down [10]. The use of a Kramers-Moyal expansion in that equation results in a corresponding Fokker-Planck equation which provides a diffusional approximation to the discrete dynamics. The corresponding Langevin equations then reads

$$
\frac{\mathrm{d}}{\mathrm{d} t} x=\alpha_{x}(V)(1-x)-\beta_{x}(V) x+\xi_{x}(t), \quad x=m, h, n .
$$

Here, $\xi_{x}(t)$ are independent Gaussian white noise sources of vanishing mean. For an excitable membrane patch with $N_{\mathrm{Na}}$ sodium and $N_{\mathrm{K}}$ potassium ion channels, the noise correlations assume the following form:

$$
\left\langle\xi_{m}(t) \xi_{m}\left(t^{\prime}\right)\right\rangle=\frac{1}{N_{\mathrm{Na}}}\left[\alpha_{m}(V)(1-m)+\beta_{m}(V) m\right] \delta\left(t-t^{\prime}\right),
$$




$$
\begin{aligned}
& \left\langle\xi_{h}(t) \xi_{h}\left(t^{\prime}\right)\right\rangle=\frac{1}{N_{\mathrm{Na}}}\left[\alpha_{h}(V)(1-h)+\beta_{h}(V) h\right] \delta\left(t-t^{\prime}\right), \\
& \left\langle\xi_{n}(t) \xi_{n}\left(t^{\prime}\right)\right\rangle=\frac{1}{N_{\mathrm{K}}}\left[\alpha_{n}(V)(1-n)+\beta_{n}(V) n\right] \delta\left(t-t^{\prime}\right) .
\end{aligned}
$$

The stochastic equation (5) replaces equation (4). Note that the correlations of the stochastic forces in these Langevin equations contain the corresponding state-dependent variables and thus should be Itô-interpreted because it is derived in a diffusional approximation of a jump process which is intrinsically a white noise problem [37-40], and does not result from a white noise limit of a colored noise problem [41].

Within the approximation of homogeneous ion channel densities, $\rho_{\mathrm{Na}}=60 \mu \mathrm{m}^{-2}$ and $\rho_{\mathrm{K}}=18 \mu \mathrm{m}^{-2}$, the ion channel numbers are given by $N_{\mathrm{Na}}=\rho_{\mathrm{Na}} S, \quad N_{\mathrm{K}}=\rho_{\mathrm{K}} S$, with $S$ being the size of the membrane patch. The number of ion channels, or the size of the excitable membrane patch $S$, determines the strength of the fluctuations and thus the channel noise level. With a decreasing patch size, i.e. decreasing number of ion channels, the noise level caused by fluctuations of the number of open ion channels increases, cf equations $(6 a)$ $(6 c)$. Within this modeling, the rate for spontaneous spiking depends monotonically on the patch size $[15,21,25]$.

\section{Gating charges and currents in a modified Hodgkin-Huxley model}

Our starting point for the derivation of the gating charge values are the opening and closing rates, equations $(3 a)$ $(3 f)$, of the standard $\mathrm{HH}$ model. Conformational changes of the ion channel in order to switch between open and closed configurations come along with the movement of gating charge [1, 27]. Assuming Arrhenius-like dependences, the transition rates are [27]

$$
\begin{gathered}
\alpha(V)=\alpha_{0} \exp \left\{\frac{q z \cdot V}{k_{B} T}\right\}, \\
\beta(V)=\beta_{0} \exp \left\{\frac{-q(1-z) \cdot V}{k_{B} T}\right\},
\end{gathered}
$$

containing the voltage-independent parts $\alpha_{0}, \beta_{0}$, the gating charge $q$, and the asymmetry parameter $z$ with $z \in(0,1)$. The two latter parameters can be deduced by a comparison of equations $(7 a)-(7 b)$ with equations $(3 a)-(3 f)$ in the high activation barrier limit, being assumed at large negative voltage values [42, 43], yielding

$$
\begin{aligned}
& \alpha_{n}(V) \propto \exp \{+V / 10\}, \\
& \beta_{n}(V) \propto \exp \{-V / 80\}, \\
& \alpha_{h}(V) \propto \exp \{-V / 20\}, \\
& \beta_{h}(V) \propto \exp \{+V / 10\}, \\
& \alpha_{m}(V) \propto \exp \{+V / 10\}, \\
& \beta_{m}(V) \propto \exp \{-V / 18\} \text {. }
\end{aligned}
$$

The corresponding gating charges then follow as

$$
\begin{gathered}
q_{n}=2.709 e, \\
q_{h}=-3.612 e, \\
q_{m}=3.746 e,
\end{gathered}
$$

with the elementary charge $e=1.6022 \times 10^{-19}$ A s. In particular, $q_{m}$ and $q_{n}$ correspond to motion of effective positive charges during the gate openings [27] from the interior side of cell membrane to the exterior one (outward motion). The total gating charges are

$$
\begin{gathered}
Q_{\mathrm{K}}^{n \text {-charge }}=q_{n} \times 4 N_{\mathrm{K}}, \\
Q_{\mathrm{Na}}^{\mathrm{m} \text {-charge }}=q_{m} \times 3 N_{\mathrm{Na}}, \\
Q_{\mathrm{Na}}^{\mathrm{h} \text {-charge }}=q_{h} \times 1 N_{\mathrm{Na}} .
\end{gathered}
$$

To obtain the gating currents, one has to multiply the gating charges with time derivatives of the corresponding gating variables. Adding the corresponding current densities (per unit area) to the equation for voltage variable (1), we find

$$
\begin{gathered}
I_{\mathrm{ext}}(t)=C \frac{\mathrm{d} V(t)}{\mathrm{d} t}+g_{\mathrm{Na}}^{\max } m^{3} h\left(V-V_{\mathrm{Na}}\right) \\
+g_{\mathrm{K}}^{\max } n^{4}\left(V-V_{\mathrm{K}}\right)+g_{l}\left(V-V_{l}\right) \\
+\rho_{\mathrm{Na}} 3 \frac{\mathrm{d} m}{\mathrm{~d} t} q_{m}+\rho_{\mathrm{Na}} \frac{\mathrm{d} h}{\mathrm{~d} t} q_{h}+\rho_{\mathrm{K}} 4 \frac{\mathrm{d} n}{\mathrm{~d} t} q_{n},
\end{gathered}
$$

where $\rho_{\mathrm{X}}$ are the specific ion channel densities. The dynamics of the gating variables are given by equation (5) and equations $(6 a)-(6 c)$. Taken together, all these equations constitute a stochastic generalization of the $\mathrm{HH}$ model which accounts for gating current effects being considered in the present work for the first time.

\subsection{Deterministic case: neglecting channel noise}

In order to study the influence of gating charge on the generation of action potentials in more detail we consider the case of constant current driving, i.e. $I_{\text {ext }}(t)=$ const. Figure 1 depicts the bifurcation scenario for spike occurrence in the original $\mathrm{HH}$ model and in the generalized model which takes gating charge into account. As, for the moment, we want to neglect channel noise (which corresponds to the limit of infinite patch size or infinite numbers of ion channels), the gating dynamics, equation (5), reduces to that in equation (4).

While the existence of the fixed point solution is not affected by the gating charge effects, both its stability range and the emergence of the oscillatory spiking solution are shifted toward larger values of the external driving current, cf figure 1(a). This again verifies the inhibitory functionality of the gating charge. For the present case, the critical current value at which the fixed point loses stability is $I_{\text {ext }} \approx 10.81 \mu \mathrm{Acm}^{-2}$, whereas in the standard $\mathrm{HH}$ model it occurs at $I_{\mathrm{ext}} \approx 9.76 \mu \mathrm{A} \mathrm{cm}^{-2}[44,45]$. Yet, before this happens in both models in (1) and (11), a periodic, spiking solution emerges via a subcritical Hopf bifurcation. Then both the fixed point solution and the limit cycle, i.e. the spiking solution, coexist for a certain range of driving currents. The periods of the solutions are depicted in figure 1 $(b)$. The spiking period versus the driving current is also shifted toward higher values of the driving current.

Figure 1 demonstrates that the gating charge does not affect the rest potential. It impedes, however, the system's excitability. For a given $I_{\text {ext }}$ the threshold for excitation is 

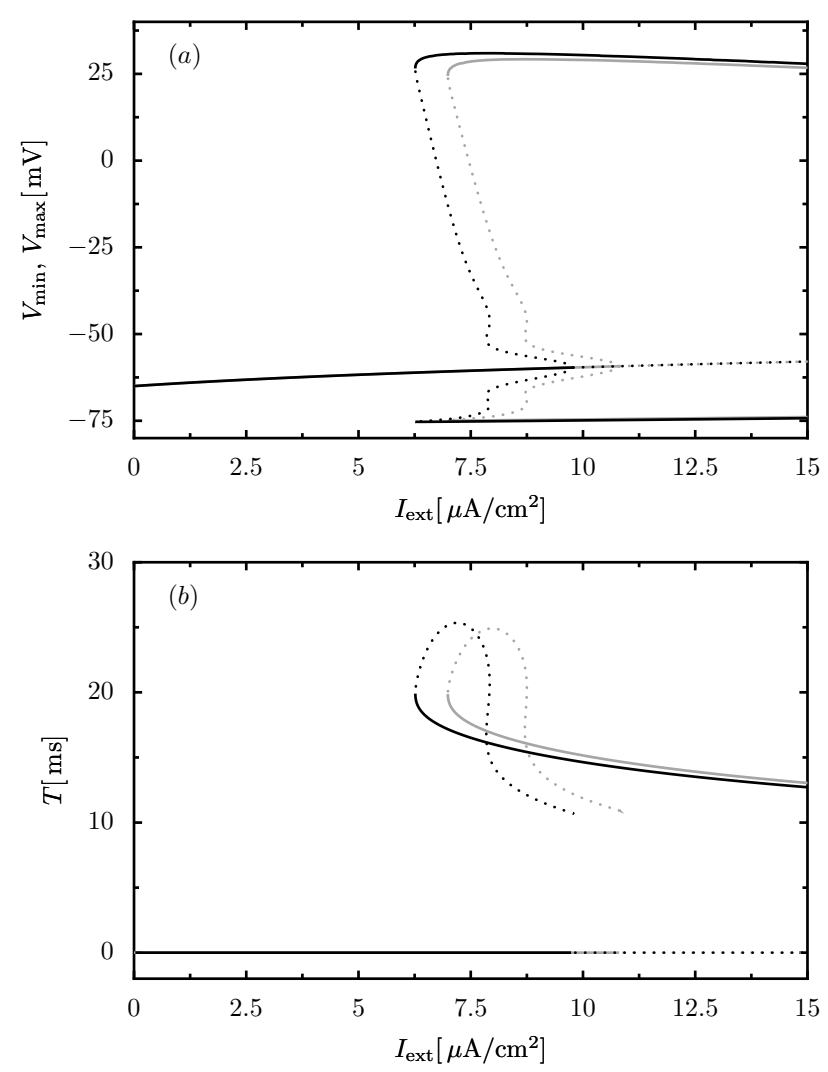

Figure 1. The bifurcation diagrams for the original deterministic $\mathrm{HH}$ model in (1) (black lines) and the deterministic generalized model (gray lines) in (11), which takes gating charge effects into account, are plotted versus the external constant driving current $I_{\text {ext }}$. The continuous lines correspond to stable solutions; the dotted lines refer to the unstable solutions. In $(a)$ the minimal and maximal membrane potentials are plotted. In $(b)$ the period of the spiking is depicted. A horizontal line at zero indicates the existence of a non-spiking solution.

increased, meaning that the excitability in the case of a gating charge dynamics becomes decreased as compared to the case when no gating charge effects are present. In addition, the time period of an oscillatory spiking solution is increased.

\subsection{Deterministic case: increment of the membrane time constant}

At the beginning of an excitation the $m$ gates open, therefore $\frac{\mathrm{d} m}{\mathrm{~d} t}>0$. Consequently, the $m$-gating current is positive (corresponding to an outward motion of positive charges), i.e. $I_{\mathrm{Na}}^{\mathrm{m} \text {-gating }}=3 N_{\mathrm{Na}} q_{m} \frac{\mathrm{d} m}{\mathrm{~d} t}>0\left(q_{m}>0\right)$. The opening of sodium channels enables transport of positively charged sodium ions into the cytoplasm (implying a negative current by standard convention). Thus, these two currents (the gating or the polarization current stemming from the ion channels and the ion current) are oppositely directed. At the very beginning of the spike initiation (the gating variable $m$ is about 0.1 at the threshold), the absolute value of the gating current is around $10 \%$ of the ionic current. The reason is that the gating current is proportional to the $m$ variable, whereas the averaged ion current scales with $\mathrm{m}^{3}$. Moreover, the gating current enhances the overall polarization current across the membrane. As a result, the membrane time constant becomes effectively enhanced as it can be deduced from the following reasoning: to study the spike initiation, only the fastest gating variable $m(t)$, which rapidly adjusts to the steady state value, $m_{\infty}(V)=\alpha_{m}(V) /\left[\alpha_{m}(V)+\beta_{m}(V)\right]$ should be taken into account. Note also that $n(t)$ and $h(t)$ exhibit slow dynamics, and their values can be taken at the rest potential $V_{\text {rest }}=-65.0 \mathrm{mV}$. It then follows, using $\frac{\mathrm{d} m(V(t))}{\mathrm{d} t} \approx \frac{\mathrm{d} m_{\infty}(V)}{\mathrm{d} V} \frac{\mathrm{d} V}{\mathrm{~d} t}$, that the influence of gating currents can be accounted for by a voltage-dependent contribution to the membrane capacitance. Consequently, taking this $m$-gating charge contribution into account, the effective membrane capacitance reads $C_{\text {eff }}(V)=C+C_{g}(V)$, where

$$
\begin{aligned}
C_{g}(V) & =3 \rho_{\mathrm{Na}} q_{m} \frac{\mathrm{d} m_{\infty}(V)}{\mathrm{d} V} \\
& =3 \rho_{\mathrm{Na}} q_{m} m_{\infty}(V)\left[1-m_{\infty}(V)\right] \frac{\mathrm{d}}{\mathrm{d} V} \ln \frac{\alpha_{m}(V)}{\beta_{m}(V)} .
\end{aligned}
$$

This latter relation assumes a simple and insightful form in the approximation of equations $(7 a),(7 b)$ :

$$
C_{g}(V) \approx 3 \rho_{\mathrm{Na}} \frac{q_{m}^{2}}{k_{B} T} m_{\infty}(V)\left[1-m_{\infty}(V)\right] .
$$

This expression evidences that $C_{g}(V)$ is maximal when the sodium $m$-gates are half-open, i.e. $m_{\infty}=1 / 2$. For $q_{m}$ given in equation $(9 c)$ and with $T=6.3{ }^{\circ} \mathrm{C}$, we have $C_{g} \approx$ $2.8 \times 10^{-16} \rho_{\mathrm{Na}} m_{\infty}(V)\left[1-m_{\infty}(V)\right]$ F. Furthermore, for $\rho_{\mathrm{Na}}=60 \mu \mathrm{m}^{-2}$, and for $m_{\infty} \approx 0.05$ at the resting potential, $C_{g}\left(V_{\text {rest }}\right) \approx 0.085 \mu \mathrm{F} \mathrm{cm}^{-2}$, i.e. the additional capacitance loading caused by sodium channels amounts to about $8.5 \%$ of the bare membrane capacitance at the rest potential. However, it can transiently be almost $42 \%$ of the bare capacitance when one-half of the sodium gates is open. The membrane time constant at rest $\tau_{\text {rest }}=C_{\text {rest }} / G_{\text {rest }}$, where $G_{\text {rest }}$ is the corresponding membrane slope conductance, is increased accordingly. Therefore, the spiking dynamics slows down, cf figure $1(b)$.

\section{Channel noise and gating charge effects}

The behavior predicted by the deterministic model should also carry implications for the spontaneous spiking in the absence of stimulus, i.e. $I_{\text {ext }}=0$. Especially, we expect a reduction of the spiking activity due to gating charge effects for membrane patches of a finite size. This indeed is the case as we shall demonstrate below.

One of the major consequences of intrinsic channel noise is the initiation of spontaneous action potentials $[3,6-9,11]$. A quantitative measure for the occurrence of the action potentials is the mean interspike interval, i.e.

$$
\langle T\rangle=\frac{1}{N} \sum_{i=1}^{N}\left(t_{i}-t_{i-1}\right),
$$

where $t_{i}, i=1, \ldots, N$, are the times for the occurrence of spike events and we set $t_{0}=0$. Since the strength of the channel noise depends on the size of the membrane patch, $\langle T\rangle$ is a function of the patch size $S$. With an increasing noise level 


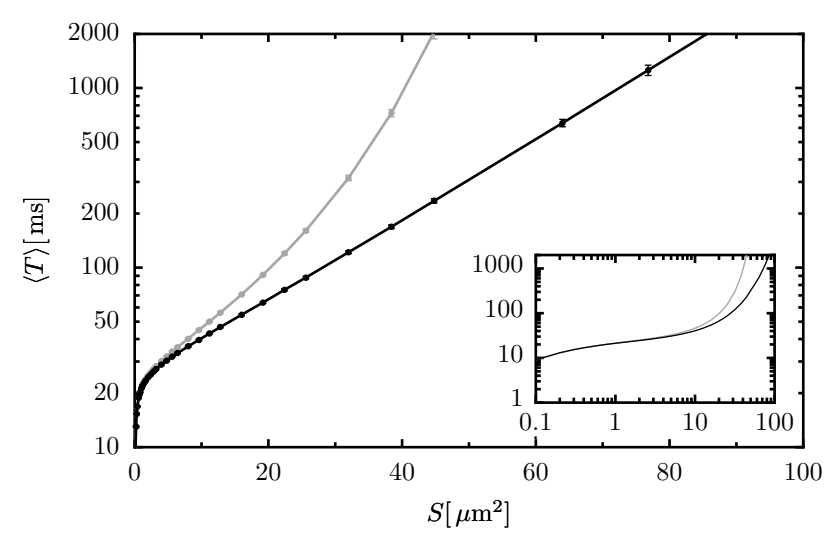

Figure 2. The dependence of the mean interspike interval $\langle T\rangle$ on the size $S$ of the membrane patch is depicted. The accuracy of the numerical simulation results is indicated by the error bars. If gating currents are considered, the spontaneous spiking activity, see the gray line, is reduced compared to the case for which gating charge effects are neglected, as indicated by the black line. Especially, for large patch sizes or large numbers of ion channels, gating charge effects lead to a drastically reduced spontaneous spiking activity. In the inset, we depict the same data on the log-log scale.

or decreasing patch sizes $S$, the spike production increases and thus the mean interspike interval $\langle T\rangle$ decreases and even can approach the refractory time $[15,16]$.

The spike occurrences $t_{i}$ are extracted from the voltage train $V(t)$ which we obtain from a numerical integration of the generalized HH model, cf equations (11), (5) and (6a)$(6 c)$. The integration is carried out by a standard stochastic Euler algorithm with a step size of $1 \mu \mathrm{s}$. The Gaussian random numbers are generated by the 'numerical recipes' routine ran2 using the Box-Muller algorithm [46]. To ensure the confinement of the gating variables between 0 (all gates are closed) and 1 (all gates are open), we have implemented numerically reflecting boundaries at 0 and 1 . The occurrences of action potentials are determined by upward crossings of the membrane potential $V$ of a certain detection threshold. Due to the very steep increase of membrane potential at firing, the actual choice of the detection threshold does not affect the results. For each trajectory, we performed $2^{28}$ simulation time steps. Moreover, an ensemble averaging over 100 different trajectories was done.

In figure 2 we depict the dependence of the mean interspike interval $\langle T\rangle$ on the patch size $S$. Due to the inhibitory nature of the gating charge, the mean interspike interval for a given membrane patch is significantly increased as compared to the case where gating charge effects are neglected. This leads to a diminishing strength of the channel noise component, mostly responsible for stochastic self-excitation. To put it differently, stochastic fluctuations of the membrane capacitance partially compensate the channel noise effect. Because the mean interspike interval is exponentially sensitive to the channel noise intensity, even a small relative reduction of noise intensity results in an exponentially large effect for large membrane patches. Therefore, the spontaneous spiking activity is drastically reduced. For example, for patch sizes around $45 \mu \mathrm{m}^{2}$ the probability of an occurrence of a

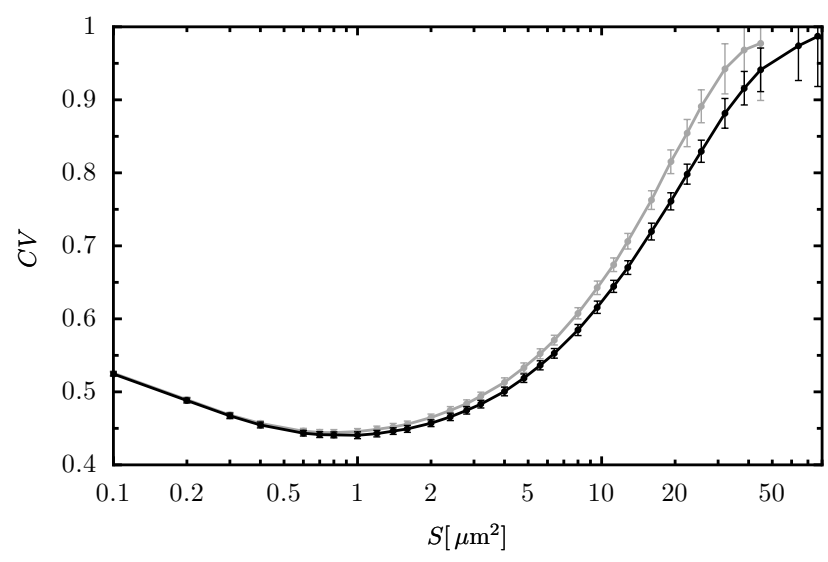

Figure 3. Same stochastic data as in figure 2, but evaluated for the coefficient of variation $C V$; cf equation (15).

spontaneous action potential is one order of magnitude less in the case of gating charge effects as compared to the case when gating charge effects are neglected. For larger patch sizes, the discrepancy becomes ever more striking. In clear contrast, the gating charge effects can safely be neglected for very small patch sizes for which the channel noise rules the dynamics. Note that even though in this region a discrete stochastic modeling is more appropriate, recent results show that the Langevin approach does work quite well also for small patch sizes [23].

\section{Coherence of spontaneous spiking activity}

Next we investigate the influence of the gating charge on the regularity of the spontaneous spiking. A proper measurement is provided by the coefficient of variation, $C V$, which is given as the ratio of standard deviation to the mean value of the interspike intervals, i.e.

$$
C V=\frac{\sqrt{\left\langle T^{2}\right\rangle-\langle T\rangle^{2}}}{\langle T\rangle}
$$

with the mean squared interspike interval given by $\left\langle T^{2}\right\rangle:=$ $\frac{1}{N} \sum\left(t_{i}-t_{i-1}\right)^{2}$. For a fully disordered point process, like a Poisson process, the coefficient of variation $C V$ assumes the value $C V=1$. For a more ordered process $C V$ attains a smaller value, and for a deterministic signal it vanishes.

Previous studies [15, 16], where such gating current effects have been neglected, have demonstrated that the $C V$ exhibits a distinct minimum for an optimal patch size $S \approx 1 \mu \mathrm{m}^{2}$. In our case again, the spiking is mostly regular at almost the same value, cf figure 3 . This phenomenon has been termed intrinsic coherence resonance [15, 47, 48]. In figure 3, the coefficient of variation is plotted versus the patch sizes for the two cases, with and without the gating charge effect considered; the $C V$ shows a distinct minimum for both cases. Though the most regular spiking could be observed at almost the same patch size, namely $S=1 \mu \mathrm{m}^{2}$, the spiking coherence slightly deteriorates due to the gating currents' influence. The main effect of gating charge on stochastic dynamics is thus to 
slow down the spiking activity and this effect can be essential, cf figure 2 .

\section{Conclusion and outlook}

We extended a stochastic description of the $\mathrm{HH}$ model accounting for inherent channel noise by including gating current effects, which equivalently can be described in terms of capacitance fluctuations; see in equation (12). Our study revealed that while the deterministic $\mathrm{HH}$ model with gating charge effects does not differ dramatically from the original model for the standard set of parameters, the corresponding stochastic model does behave very differently for intermediateto-large membrane patch sizes. A main finding is that spontaneous spiking activity becomes drastically reduced. The physical reason of this is that the gating current of translocated gating charges in sodium channels is counter-directed to the electrical current of sodium ions, finally leading to excitation after the whole system is driven by external current beyond a threshold. This results in the reduced channel noise intensity. Simultaneously, there occurs a rise of the membrane time constant resulting in longer spiking periods for supra-threshold driving.

For the parameters studied, i.e. for the original $\mathrm{HH}$ parameters, the effects are relatively small in the noiseless case. Nevertheless, even deterministically, gating charge effects ultimately lead to the existence of optimal ion channel densities for neuronal information transduction [31, 32]. Notably, an increase of the sodium channel density by a factor of 20 (as in mammalian Ranvier nodes) would cause gating charge effects of increased importance. The striking feature revealed in this work is that the gating charge effects can play a fundamental role in the spontaneous spiking dynamics of intermediateto-large membrane patches even if they are still relatively minor deterministically. The reduction of spontaneous spiking exceeds one order of magnitude starting from a patch size around $45 \mu \mathrm{m}^{2}$ for the parameters studied. This provides an inherent mechanism for the channel noise reduction in neurons. This might explain the discrepancy between the theoretical predictions of a stochastic $\mathrm{HH}$ model and some experimental results for real neurons, e.g. see [49]. However, the effect of intrinsic coherence resonance as a signature of the influence of channel noise in excitable membranes still remains.

We share the confident belief that our investigations of the influence of gating charge effects on the channel noise-induced spiking activity in an archetypal model provide some new insights into the underlying physical principles and mechanism of neuronal signaling.

\section{Acknowledgments}

This work has been supported by the Deutsche Forschungsgemeinschaft via the Sonderforschungsbereich SFB-486, project A10.

\section{Glossary}

Coherence resonance. Noise-induced improvement of the regularity of the system's output. If the noise stems from internal dynamics, the effect is called intrinsic coherence resonance.

Hopf-bifurcation. A change from a topology with a fixed point solution to a topology with an oscillatory solution under a small variation of a parameter within a nonlinear system.

Itô-Stratonovich dilemma. Interpretation problem which arises in the context of Langevin equations in the case of multiplicative Gaussian white noise.

Kramers-Moyal expansion. Procedure to transform a master equation for a discrete stochastic process into a Fokker-Planck equation for an approximate continuous stochastic process.

Poisson process. A stochastic process which is memoryless (Markovian) with exponentially distributed waiting times between two successive events.

Stochastic resonance. An anomalous, noise-assisted enhancement of transduction of weak (deterministic or stochastic) signals.

\section{References}

[1] Hodgkin A L and Huxley A F 1952 A quantitative description of membrane current and its application to conduction and excitation in nerve J. Physiol. 117500

[2] Lecar H and Nossal R 1971 Theory of threshold fluctuations in nerves Biophys. J. 111048

[3] White J A, Rubinstein J T and Kay A R 2000 Channel noise in neurons Trends Neurosci. 23131

[4] Koch K 1999 Biophysics of Computation: Informational Processing in Single Neurons (New York: Oxford University Press)

[5] van Rossum M C W, O'Brien B J and Smith R G 2003 Effects of noise on the spike timing precision of retinal ganglion cells J. Neurophys. 892406

[6] Skaugen E and Walløe L 1979 Firing behavior in a stochastic nerve membrane model based upon the Hodgkin-Huxley equtions Acta Physiol. Scand. 107343

[7] Clay J R and DeFelice L J 1983 Relationship between membrane excitability and single channel open-close kinetics Biophys. J. 42151

[8] Strassberg A F and DeFelice L J 1993 Limitations of the Hodgkin-Huxley formalism-effects of single-channel kinetics on transmembrane voltage dynamics Neural Comput. 5843

[9] DeFelice L J and Isaac A 1993 Chaotic states in a random world-relationship between the nonlinear differentialequations of excitability and the stochastic properties of ion channels J. Stat. Phys. 70339

[10] Fox R F and Lu Y 1994 Emergent collective behavior in large numbers of globally coupled independently stochastic ion channels Phys. Rev. E 493421

[11] Chow C C and White J A 1996 Spontaneous action potentials due to channel fluctuations Biophys. J. 713013

[12] Schneidman E, Freedman B and Segev I 1998 Ion channel stochasticity may be critical in determining the reliability and precision of spike timing Neuronal Comput. 101679 
[13] Bezrukov S M and Vodyanoy I 1995 Noise-induced enhancement of signal-transduction across voltage-dependent ion channels Nature $\mathbf{3 7 8} 362$

[14] Bezrukov S M and Vodyanoy I 1997 Signal transduction across alamethicin ion channels in the presence of noise Biophys. J. 732456

[15] Schmid G, Goychuk I and Hänggi P 2001 Stochastic resonance as a collective property of ion channel assemblies Europhys. Lett. 5622

[16] Jung P and Shuai J W 2001 Optimal sizes of ion channel clusters Europhys. Lett. 5629

[17] Gammaitoni L, Hänggi P, Jung P and Marchesoni F 1998 Stochastic resonance Rev. Mod. Phys. 70223

[18] Hänggi P 2002 Stochastic resonance in biology — how noise can enhance detection of weak signals and help improve biological information processing Chem. Phys. Chem. 3285

[19] Shuai J W and Jung P 2003 Optimal ion channel clustering for intracellular calcium signaling Proc. Natl Acad. Sci. 100506

[20] Ginzburg S L and Pustovoit M A 2003 Bursting dynamics of a model neuron induced by intrinsic channel noise Fluct. Noise Lett. L 3265

[21] Schmid G, Goychuk I, Hänggi P, Zeng S and Jung P 2004 Stochastic resonance and optimal clustering for assemblies of ion channels Fluct. Noise Lett. 4 L33

[22] Gong Y B, Wang M S, Hou Z H and Xin H W 2005 Optimal spike coherence and synchronization on complex HodgkinHuxley neuron networks Chem. Phys. Chem. 61042

[23] Shuai J W and Jung P 2006 The dynamics of small excitable ion channel clusters Chaos 16026104

[24] Ya P L and Qian S L 2006 Implicit and explicit internal signal stochastic resonance in calcium ion oscillations $\mathrm{Chem}$. Phys. Lett. 417498

[25] Schmid G, Goychuk I and Hänggi P 2003 Channel noise and synchronization in excitable membranes Physica A 325165

[26] Schmid G, Goychuk I and Hänggi P 2004 Effect of channel block on the spiking activity of excitable membranes in a stochastic Hodgkin-Huxley model Phys. Biol. 161

[27] Hille B 2001 Ionic Channels of Excitable Membranes 3rd edn (Sunderland, MA: Sinauer Associates)

[28] Armstrong C M and Bezanilla F 1973 Currents related to movement of gating particles of sodium channels Nature 242459

[29] Keynes R D and Rojas E 1973 Characteristics of sodium gating current in squid giant axon J. Physiol. $23328 \mathrm{P}$

[30] Armstrong C M and Bezanilla F 1974 Charge movement associated with opening and closing of activation gates of Na channels J. Gen. Physiol. 63533
[31] Hodgkin A 1975 Optimum density of sodium channels in an unmyelinated nerve Philos. Trans. R. Soc. Lond. 270297

[32] Adrian R H 1975 Conduction-velocity and gating current in squid giant-axon Proc. R. Soc. 18981

[33] Hudspeth A J, Choe Y, Mehta A D and Martin P 2000 Putting ion channels to work: mechanoelectrical transduction, adaptation, and amplification by hair cells Proc. Natl Acad. Sci. 9711765

[34] Nossal R J and Lecar H 1991 Molecular and Cell Biophysics (Redwood City, CA: Addison-Wesley)

[35] Adair R K 2004 Optimum ion channel properties in the squid giant axon Phys. Rev. E 69042902

[36] Sangrey T D, Friesen W O and Levy W B 2004 Analysis of the optimal channel density of the squid giant axon using a reparameterized Hodgkin-Huxley model J. Neurophysiol. 912541

[37] Hänggi P 1980 Langevin description of Markovian integro-differential master-equations Z. Phys. B 36271

[38] Hänggi $P 1981$ Langevin description of Markov master-equations: II. Noise correlations Z. Phys. B 43269

[39] Hänggi P and Thomas H 1982 Stochastic processes-time evolution, symmetries and linear response Phys. Rep. 88207 see in section 6.2

[40] Zwanzig R 2001 A chemical Langevin equation with non-Gaussian noise J. Phys. Chem. B 1056472

[41] Hänggi P and Jung P 1995 Colored noise in dynamical-systems Adv. Chem. Phys. 89239

[42] Goychuk I and Hänggi P 2002 Ion channel gating: a first-passage time analysis of the Kramers type Proc. Natl Acad. Sci. 993552

[43] Goychuk I and Hänggi P 2003 The role of conformational diffusion in ion channel gating Physica A 3259

[44] Hassard B 1978 Bifurcation of periodic-solutions of Hodgkin-Huxley model for squid giant-axon J. Theor. Biol. 71401

[45] Rinzel J and Miller R N 1980 Numeric-calculation of stable and unstable periodic-solutions to the Hodgkin-Huxley equations Math. Biosci. 4927

[46] Press W H, Teukolsky S A, Vetterling W T and Flannery B P 1992 Numerical Recipes in C 2nd edn (Cambridge: Cambridge University Press)

[47] Pikovsky A S and Kurths J 1997 Coherence resonance in a noise-driven excitable system Phys. Rev. Lett. 78775

[48] Lindner B, Garcia-Ojalvo J, Neiman A and Schimansky-Geier L 2004 Effects of noise in excitable systems Phys. Rep. 392321

[49] Diba K, Lester H A and Koch Ch 2004 Intrinsic noise in cultured hippocampal neurons: experiment and modeling J. Neurosci. 249723 